\section{Contact modelling of human skin: What value to use for the modulus of elasticity?}

\author{
Julien van Kuilenburg, Marc A Masen and Emile van der Heide
}

Proc IMechE Part J:

J Engineering Tribology 227(4) 349-361

(C) University of Twente 2012

Reprints and permissions:

sagepub.co.uk/journalsPermissions.nav DOI: $10.1177 / 1350650112463307$ pij.sagepub.com

\begin{abstract}
In modelling and understanding the contact and friction behaviour of human skin, the elastic modulus of the skin is an important input parameter. For the development of design rules for the engineering of surfaces in contact with the skin an expression that describes the relation between the elastic modulus of the skin and the size of the contact is essential. Although an exact description of the mechanical behaviour of the skin requires an anisotropic, nonlinear, viscoelastic model, in this study it was found that for contact modelling involving relatively small deformations, the mechanical behaviour seems to be accurately described by a single parameter: the effective elastic modulus. The effective elastic modulus is shown to decrease several orders of magnitude when the length scale increases, which is the consequence of the rather complex anatomy of the skin. At an indentation depth of $10 \mu \mathrm{m}$, the effective elastic modulus was shown to decrease from 0.15 to $0.015 \mathrm{MPa}$ when the radius of curvature of the indenter increases from $10 \mu \mathrm{m}$ to $10 \mathrm{~mm}$. The variation of the elasticity is explained by the variation in the composition and properties of the different skin layers. This study shows that for the contact modelling of human skin, a closed-form expression based on the anatomy of the skin exists, which yields the magnitude of the effective elastic modulus of the skin as a function of the length scale of the contact depending on variables such as age, gender and environmental conditions.
\end{abstract}

\title{
Keywords
}

Skin, mechanical properties, contact modelling, friction, tribology

Date received: 7 May 2012; accepted: 12 September 2012

\section{Introduction}

To a large extent, the functionality and comfort experienced during the use of daily life products, such as apparel, household appliances and sports equipment, are determined by the frictional behaviour of the contacts that occur with the skin; yet design diagrams or design rules for the engineering of product surfaces with prescribed friction and touch properties are missing. In the field of skin tribology, the sliding contact between surfaces and the human skin is investigated with the aim to develop such design rules. The frictional behaviour of two surfaces that are in sliding contact depends on the material combination, the micro-geometry of the surfaces, any lubricants and the environmental conditions, as well as the operational conditions of the contact. ${ }^{1}$ The friction force that occurs in the contact is a combination of the forces required to break the adhesive bonds between the two surfaces and the forces related to the deformation of the bodies in contact. The adhesion component in the friction force can be expressed as the product of the shear strength of the interface and the area of the contact. When a body that indents the skin is moved forward, work is dissipated due to the viscoelastic nature of the skin. The deformation component equals the amount of work lost by viscoelastic hysteresis per unit sliding distance, thus being proportional to the indentation of the body into the skin. In many cases, the mechanical behaviour of the skin can be assumed to be elastic, so that the contact theory developed by Hertz applies. ${ }^{2-4}$ Hertz's $^{-1}$ theory ${ }^{5,6}$ is based on the assumption that the material is homogeneous and isotropic, and is valid for relatively small deformations. The dimensions of the so-called Hertzian contact are determined by the geometrical and loading parameters and the elastic behaviour of the skin.

Department of Engineering Technology, Laboratory for Surface Technology and Tribology, University of Twente, Enschede, The Netherlands

\section{Corresponding author:}

Julien van Kuilenburg, Department of Engineering Technology, Laboratory for Surface Technology and Tribology, University of Twente, PO Box 217, 7500 AE Enschede, The Netherlands.

Email: j.vankuilenburg@utwente.nl 
In modelling and understanding the contact and friction behaviour of human skin, the elastic modulus of the skin $E$ is thus an essential input parameter. ${ }^{7,8}$ As a material, skin behaves in a complex manner; it has a layered structure with highly changing properties through the layers, its behaviour is viscoelastic, anisotropic and there may be an influence of underlying tissue and bones. The properties of the skin vary with anatomical location and with subject, age, gender, level of care and hydration. The different layers of the skin have different thicknesses and mechanical properties. The outermost surface of the skin, the stratum corneum, is a $20-40 \mu \mathrm{m}$ thick layer, which is composed of hornified cells giving this layer its high stiffness, whereas the thickness of the extremely viscous and soft hypodermis ranges from 1 to $50 \mathrm{~mm}$, depending on anatomical site and dietary habits. ${ }^{9,10}$ Common methods for quantifying the skin mechanical behaviour are extension, torsion, suction and indentation. When investigating the friction behaviour of human skin, a commonly used experimental set-up is the spherical probe sliding over the skin. At the microlevel, the contact between a rough surface and the skin can be imagined as a collection of spherical tipped asperities, each asperity indenting the skin. ${ }^{11}$ It is therefore that the indentation principle is the most appropriate technique for measuring the mechanical properties, which determines the contact and friction behaviour of human skin. Elastic moduli reported throughout the literature have been measured with a wide range of indenter geometries, making a straight translation to the particular geometry of interest difficult. Even though numerous results from experiments using spherical indenters can be found in the literature, the variation of used tip radii and normal loads does not allow an easy comparison of these values. The inner, also volar, forearm is a site commonly used for investigating tribological and mechanical properties of the skin: not only is it representative for nonglabrous skin, but it is also having a relatively low hair fiber density and its surface is relatively smooth. Figure 1 shows data for the measured elastic modulus of in vivo skin of the inner forearm obtained from the literature. Reported data for the measured elastic modulus of isolated stratum corneum as a function of indentation depth are shown in Figure 2.

Figures 1 and 2 point out that different elastic moduli should be taken into account whether modelling macrocontacts, such as skin in contact with product parts, or microcontacts, such as skin, i.e. stratum corneum, against surface features. For the purpose of contact modelling, it is of practical use to present the skin elastic properties as a function of the size of the contact. For an axisymmetric indenter this characteristic length scale can be the contact radius $a$. The appropriate value of $E$ to be used should be determined at the correct length scale and under representative conditions. A simple expression describing the relation between the elastic modulus of the skin and the size of the contact from microlevel to macrolevel is currently not available. For the development of design rules for the engineering of surfaces in contact with the skin such an expression $E=f(a)$ is essential.

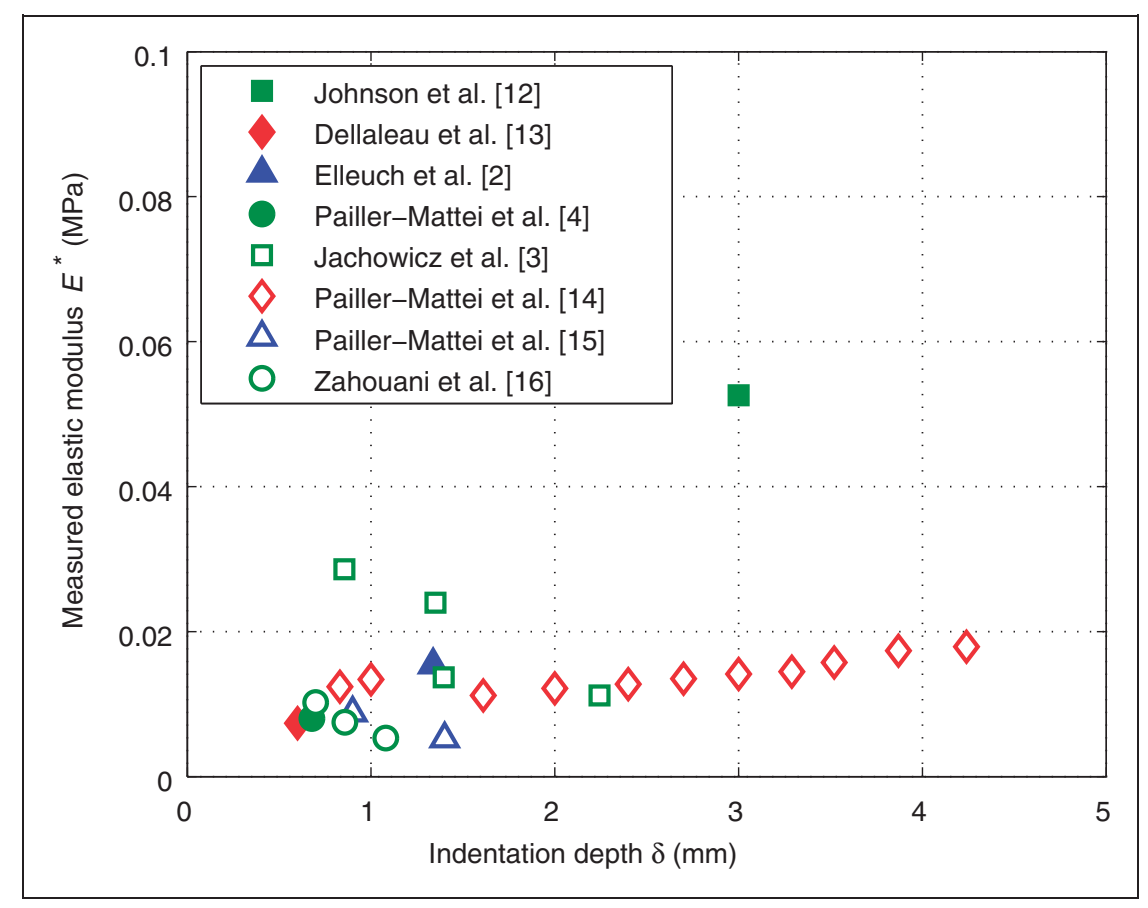

Figure I. Measured elastic modulus of in vivo skin of the human volar forearm as a function of indentation depth. Data taken from Elleuch et al., ${ }^{2}$ Jachowicz et al., ${ }^{3}$ Pailler-Mattei et al., ${ }^{4,14,15}$ Johnson et al., ${ }^{12}$ Delalleau et al.,, ${ }^{13}$ and Zahouani et al. ${ }^{16}$ 


\section{Theory}

The contact of an individual asperity is generally modelled as a spherical contact. To a first approximation, Johnson et al. ${ }^{12}$ and Adams et al. ${ }^{19}$ give the total friction force of a spherical indenter sliding against the human skin by the sum of two noninteracting terms

$$
F_{\mu}=F_{\mu, a d h}+F_{\mu, d e f}
$$

The first term in equation (1) can be calculated from the shear strength of the interface $\tau$ and the contact radius $a$ by

$$
F_{\mu, a d h}=\tau \pi a^{2}
$$

When a spherical probe indenting the skin is moved forward, work is dissipated due to the viscoelastic nature of the skin. The deformation component equals the amount of work lost by viscoelastic hysteresis per unit sliding distance, thus being proportional to a viscoelastic loss fraction $\beta$ and the indentation $\delta$ of the probe into the skin

$$
F_{\mu, d e f}=\frac{3}{16} \beta \frac{\delta}{a} F
$$

By regarding the skin as an elastic half-space loaded over a small circular region of its plane surface, the contact radius $a$ and indentation depth $\delta$ can be calculated from the normal load $F$ and radius of curvature of the body $R$ using Hertz's theory as shown in equations (4) and (5), respectively

$$
\begin{aligned}
& a=\sqrt[3]{\frac{3}{4} \frac{R F}{E^{*}}} \\
& \delta=\sqrt[3]{\frac{9}{16} \frac{F^{2}}{R E^{* 2}}}
\end{aligned}
$$

in which $E^{*}$ represents the reduced elastic modulus, defined as

$$
\frac{1}{E^{*}}=\frac{1-v_{1}^{2}}{E_{1}}+\frac{1-v_{2}^{2}}{E_{2}}
$$

with $E_{1}$ and $E_{2}$ the respective elastic moduli and $v_{1}$ and $v_{2}$ the Poisson ratios of the contacting materials. In the case of contact between skin and a stiff counterbody, $E_{\text {counterbody }} \gg E_{\text {skin }}$ and the reduced elastic modulus depends solely on the properties of the skin so that $E^{*} \approx E_{\text {skin }} /\left(1-v_{\text {skin }}^{2}\right)$. However, the skin is a layered material, being composed of three layers; the epidermis, the underlying connective tissue called the dermis and the hypodermis, or subcutis, consisting of fatty connective tissue, that connects the dermis to the underlying muscles or bones. Therefore it is questionable whether a simple elastic parameter such as the elastic modulus can be used to accurately describe the behaviour of a complex material such as the human skin.

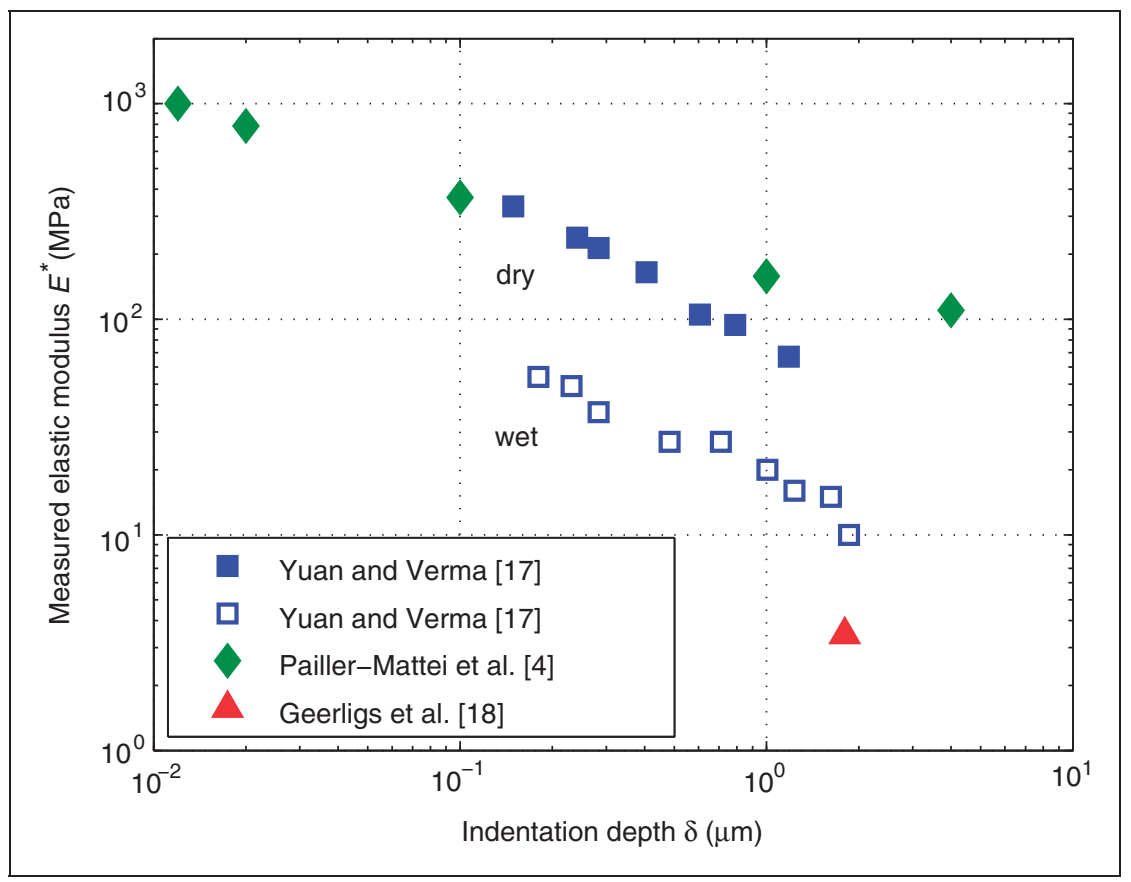

Figure 2. Measured elastic modulus of isolated stratum corneum as a function of indentation depth. Data taken from Pailler-Mattei et al., ${ }^{4}$ Yuan and Verma ${ }^{17}$ and Geerligs et al. ${ }^{18}$ 


\section{Mechanical properties of the skin}

For measuring the mechanical properties as they apply to a tribological contact in which the skin is one of the contacting surfaces, the indentation technique yields the proper results as discussed in the introduction. In an indentation experiment, an instrument continuously measures force and displacement applied to a rigid indenter. Depending on the positioning accuracy of the instrument used, mechanical properties can be determined down to the submicron level, so for skin at the level of the stratum corneum. The indentation technique can be applied in vitro on isolated skin samples or in vivo, then always measuring the combined response of the different skin layers. For any axisymmetric indenter, the reduced elastic modulus $E^{*}$ of the indented material can be obtained from the initial unloading slope of the load-displacement curve, which plots the normal load $F$ versus indentation $\delta$ as proposed by Oliver and Pharr ${ }^{20}$

$$
E^{*}=\frac{\sqrt{\pi}}{2 \sqrt{A}} \frac{\partial F}{\partial \delta}
$$

The projected area of the elastic contact $A$ can be determined from the indenter geometry and the indentation depth. For a spherical indenter, the contact radius $a$ is a function of indentation depth $\delta$ and tip radius $R$ given by Hertz as

$$
a=\sqrt{\delta R}
$$

For a conical indenter, the contact radius is related to the indentation depth through the half angle $\alpha$ of the tip so that

$$
a=\frac{2}{\pi} \frac{\delta}{\tan \alpha}
$$

For a Berkovich indenter, the projected area $A$ is obtained from the area function $A=24.5 \delta^{2}$, which relates the area to the indentation depth. ${ }^{20}$ For the purpose of scaling the results obtained with a Berkovich indenter to those obtained using an axisymmetric indenter, an equivalent contact radius is obtained from $A=\pi a^{2}$ so that $a \approx 2.8 \delta$.

A less common method to obtain the reduced elastic modulus is to fit the load-displacement data to the Hertz equation. ${ }^{3,12}$ Some studies calculate the elastic modulus by numerical analysis of the indentation curve. $^{2,13}$

\section{Stratum corneum and epidermis}

At the length scale of surface roughness, i.e. smaller than $10 \mu \mathrm{m}$, the elastic properties of the skin are determined by the top layer of the skin. Several studies report the mechanical properties of isolated stratum corneum as a function of indentation depth determined from indentation experiments, shown in Figure 2. Yuan and Verma ${ }^{17}$ obtained elastic moduli for dry and wet isolated porcine stratum corneum. Load-displacement curves were measured using a Berkovich tip. For dry stratum corneum, the reduced elastic modulus decreased from order $300 \mathrm{MPa}$ at $0.2 \mu \mathrm{m}$ indentation to about $75 \mathrm{MPa}$ at $1 \mu \mathrm{m}$. For wet stratum corneum, a considerably lower elasticity was measured decreasing from about 50 to $10 \mathrm{MPa}$ at 0.2 and $2 \mu \mathrm{m}$ indentation, respectively.

Pailler-Mattei et al. $^{4}$ compared mechanical and tribological properties of isolated stratum corneum with in vivo measurements. Isolated human stratum corneum with a thickness of around $10-20 \mu \mathrm{m}$ was attached to a glass plate. Indentation experiments were carried out using a spherical diamond tip with $7.8 \mu \mathrm{m}$ radius. By extrapolation, the elastic modulus of the skin outer surface was obtained, being around $1 \mathrm{GPa}$. The elastic modulus then decreased with increasing indentation depth to around $100 \mathrm{MPa}$ at $4 \mu \mathrm{m}$.

Geerligs et al. ${ }^{18}$ carried out indentation tests on isolated human stratum corneum and isolated human epidermis using a spherical sapphire tip with $500 \mu \mathrm{m}$ radius. For the stratum corneum, a reduced elastic modulus of $3.44 \pm 0.8 \mathrm{MPa}$ was measured at about $2 \mu \mathrm{m}$ indentation. For the epidermis, a reduced elastic modulus of $1.46 \pm 0.26 \mathrm{MPa}$ was measured at about $8 \mu \mathrm{m}$ indentation.

\section{Hydration}

Depth profiles of water content of the stratum corneum can be measured in vivo using confocal Raman spectroscopy. The moisture content of nonhydrated stratum corneum of the inner forearm can be seen to increase from below $30 \%$ at the outer surface to approximately $70 \%$ at a depth of $20 \mu \mathrm{m}$, showing a steep gradient from 5 to $15 \mu \mathrm{m} \cdot{ }^{24,25}$ Mechanical properties of the stratum corneum are strongly dependent on environmental temperature and relative humidity $(\mathrm{RH})$. Wildnauer et al. ${ }^{21}$ carried out stress-strain measurements on isolated human stratum corneum samples which were kept at different relative humidities for 48 hours. Load-elongation curves were measured on $2 \mathrm{~mm}$ wide strips. Assuming a thickness of the stratum corneum of $20 \mu \mathrm{m}$, tensile elastic moduli can be calculated decreasing from about $4 \mathrm{GPa}$ at $32 \% \mathrm{RH}$ to $2 \mathrm{GPa}$ at $76 \% \mathrm{RH}$ and $1 \mathrm{GPa}$ at $98 \%$ RH. Using a tensile tester, Park and Baddiel ${ }^{22}$ measured the tensile elastic modulus of $2 \mathrm{~mm}$ wide isolated stratum corneum strips as a function of relative humidity. The tensile elastic modulus was found to decrease from $2 \mathrm{GPa}$ at $30 \% \mathrm{RH}$ to $3 \mathrm{MPa}$ at $100 \%$ RH. Papir et al. ${ }^{23}$ investigated the influence of relative humidity and temperature on the tensile properties of isolated newborn rat stratum corneum. A continuous decrease of the elastic modulus was observed with an increase in either relative humidity 


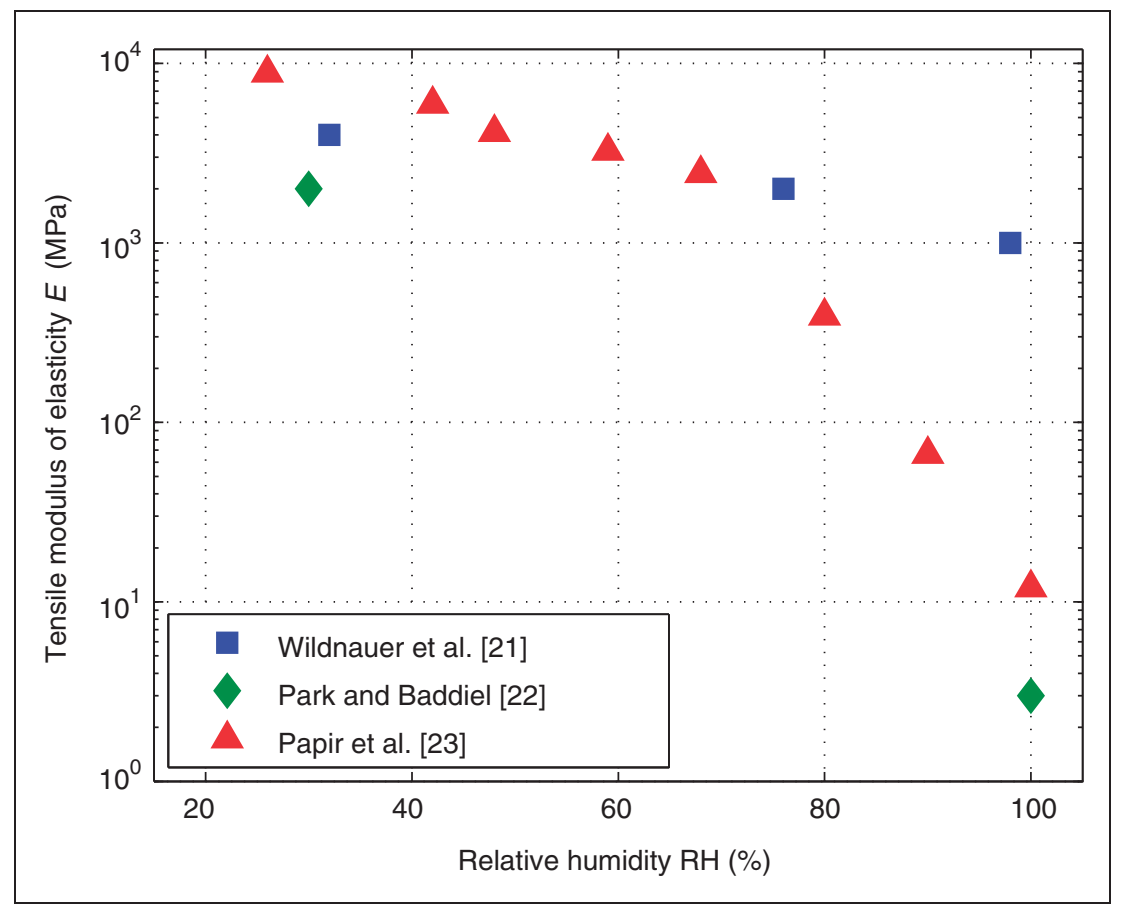

Figure 3. Tensile elastic modulus of isolated stratum corneum as a function of relative humidity. Data adapted from Wildnauer et al., ${ }^{21}$ Park and Baddiel ${ }^{22}$ and Papir et al. ${ }^{23}$

or temperature. In a semilogarithmic plot of the relative humidity versus tensile elastic modulus, two regimes can be observed separated by a clear discontinuity. At low relative humidity a moderate decline is observed from $8.87 \mathrm{GPa}$ at $26 \% \mathrm{RH}$ to $2.44 \mathrm{GPa}$ at $68 \% \mathrm{RH}$, where above $70 \% \mathrm{RH}$ an order of magnitude decrease can be seen to $12 \mathrm{MPa}$ at $100 \% \mathrm{RH}$. More recently, the influence of relative humidity on the mechanical properties of the stratum corneum has been discussed by $\mathrm{Wu}$ et al. ${ }^{26}$ They also report that their own inplane tensile measurements yielded moduli decreasing from approximately $1 \mathrm{GPa}$ to $5 \mathrm{MPa}$ with increasing hydration.

From Figure 3 it can be seen that the elasticity of the stratum corneum tends to decrease with increasing relative humidity. It is therefore important to consider temperature and humidity of the test environment when comparing the results from different experiments.

\section{Dermis and hypodermis}

At larger length scales, i.e. millimeters, the properties of the dermis and hypodermis determine the elastic behaviour of the whole skin. For example, PaillerMattei et al. ${ }^{4}$ investigated the influence of the stratum corneum on the mechanical properties of in vivo human skin by successive removal of stratum corneum layers by tape-stripping. From indentation experiments carried out on the inner forearm using a $6.35 \mathrm{~mm}$ radius indenter they found that the elastic modulus was about $7-8 \mathrm{kPa}$, independent of the thickness of the stratum corneum removed. They concluded that, at these length scales, the stratum corneum mechanical properties do not influence the global skin mechanical properties.

Delalleau et al. ${ }^{13}$ calculated the skin elastic modulus by numerical analysis of results obtained from indentation of the human forearm using a $6.22 \mathrm{~mm}$ radius spherical indenter. Isotropic, linear elastic behaviour was assumed when analysing the loadindentation curves. A reduced elastic modulus of $7.37 \mathrm{kPa}$ was measured at $600 \mu \mathrm{m}$ indentation. From numerical analysis of indentation curves measured on the inner forearm using a $6.35 \mathrm{~mm}$ radius indenter, Elleuch et al. ${ }^{2}$ obtained a reduced elastic modulus of $15.4 \mathrm{kPa}$ at $80 \mathrm{mN}$ normal load. It was observed that within the range of conditions tested, skin showed a dominant elastic behaviour.

Pailler-Mattei et al. ${ }^{15}$ compared the elastic moduli measured on the inner forearm of two subjects different in age using a $6.35 \mathrm{~mm}$ radius steel indenter. For a 30 -year-old subject a reduced elastic modulus of $8.8 \mathrm{kPa}$ was measured at $900 \mu \mathrm{m}$ indentation. At the same normal load for the 60-year-old subject, the reduced elastic modulus was $5.2 \mathrm{kPa}$ at $1400 \mu \mathrm{m}$. These results show a clear decrease of the elastic modulus with age. The same observations were made in a study by Zahouani et al. ${ }^{16}$ At $20 \mathrm{mN}$ normal load, the reduced elastic modulus decreases with age from $10.2 \mathrm{kPa}$ at 30 years to 7.5 and 5.3 $\mathrm{kPa}$ at 60 and 80 years, respectively.

\section{Variation between subjects}

Age and gender cause the effective elastic modulus to deviate from an average value. Besides age and 
gender, variation in measured mechanical properties between subjects arises from variation in skin properties such as thickness of the different skin layers or variation in aging. Differences in used test equipment, test conditions and the location on the inner forearm on which the measurements are carried out give further rise to the observed variation in results. Some studies report results obtained from a larger population of test results, so giving insight in the variation of the measured elastic properties. From an extensive study of the load-displacement response of the forearm skin, Flynn et al. ${ }^{27}$ observed a large variation within a population of 21 subjects between 21 and 52 years. Analysis of the measurement data yielded a standard deviation of about $50 \%$. Jachowicz et al. ${ }^{3}$ observed that mechanical properties changed with gender. Elastic moduli were measured on the forearm of 6 female and 4 male subjects between 28 and 65 years using a $7.94 \mathrm{~mm}$ diameter spherical probe. The reduced elastic modulus was calculated by fitting the loading part of the load-indentation curve to Hertz theory. At normal loads of 0.06 and $0.1 \mathrm{~N}$, the average reduced elastic modulus was 13.7 and $11.2 \mathrm{kPa}$ for the female subjects, where for the male subjects 28.6 and $24 \mathrm{kPa}$ were measured.

The data reported in the studies described earlier were obtained from experiments carried out on subjects of different age and gender as shown in Table 1. From the results reported in the literature it is evident that the subject age. ${ }^{15,16}$ and gender ${ }^{3}$ have a considerable influence on the measured elastic properties.

\section{Subcutaneous tissue}

With the length scale increasing further, the influence of underlying tissues such as muscles and bones becomes apparent. Pailler-Mattei et al. ${ }^{14}$ measured the elastic properties of the skin as a function of indentation depth using a conical steel indenter with $45^{\circ}$ half angle and $10 \mathrm{~mm}$ height. Elastic moduli were

Table I. Variation between subjects.

\begin{tabular}{|c|c|c|c|}
\hline Study & Subjects & Age & $\begin{array}{l}\text { Data elastic } \\
\text { modulus }\end{array}$ \\
\hline \multirow[t]{4}{*}{ Jachowicz et al. $^{3}$} & 4 males & 28-65 years & $28.6 \pm 4 \mathrm{kPa}$ \\
\hline & & & $24 \pm 3.8 \mathrm{kPa}$ \\
\hline & 6 females & $28-65$ years & $\mathrm{I} 3.7 \pm 5.3 \mathrm{kPa}$ \\
\hline & & & $1 \mathrm{I} .2 \pm 2.8 \mathrm{kPa}$ \\
\hline $\begin{array}{l}\text { Pailler-Mattei } \\
\text { et al. }{ }^{14}\end{array}$ & 10 males & about 30 years & $18 \pm 4 \mathrm{kPa}$ \\
\hline \multirow{2}{*}{$\begin{array}{l}\text { Pailler-Mattei } \\
\text { et al. }{ }^{15}\end{array}$} & I female & 30 years & $8.8 \mathrm{kPa}$ \\
\hline & I female & 60 years & $5.2 \mathrm{kPa}$ \\
\hline Zahouani et al. ${ }^{16}$ & 20 females & $55-70$ years & $8.3 \pm 1.74 \mathrm{kPa}$ \\
\hline \multirow[t]{2}{*}{ Geerlings et al. ${ }^{18}$} & 3 females & $43 \pm 4$ years & $3.44 \pm 0.8 \mathrm{MPa}$ \\
\hline & & & $\mathrm{I} .46 \pm 0.26 \mathrm{MPa}$ \\
\hline
\end{tabular}

calculated from the normal contact stiffness obtained from the initial part of the unloading curve. From about $1.5 \mathrm{~mm}$ depth, the elastic modulus increases with indentation depth from 12 to $18 \mathrm{kPa}$ at about $4 \mathrm{~mm}$. It was stated that this increase is due to the increasing contribution of subcutaneous tissues such as the muscles. From data found in the literature by Pailler-Mattei et al., ${ }^{14}$ a reduced elastic modulus for muscle was calculated varying between 32 and $96 \mathrm{kPa}$, which is larger than the elasticity measured for whole skin. Below $1.5 \mathrm{~mm}$ indentation, the elastic modulus tends to increase with decreasing indentation.

In a pioneering study on the tribology of human skin, Johnson et al. ${ }^{12}$ investigated the sliding contact of a glass probe on the inner forearm under different conditions. The mechanical response of the skin to normal loading was measured for a glass sphere with $8 \mathrm{~mm}$ radius of curvature. The loading curve followed a power law with an exponent of 1.44 , very close to the Hertz equation (5). At $1 \mathrm{~N}$ normal load and $3 \mathrm{~mm}$ indentation, a reduced elastic modulus of $52.6 \mathrm{kPa}$ was calculated using Hertz's theory. Iivarinen et al. ${ }^{28}$ obtained elastic moduli of the skin from numerical analysis of the results from experiments using a $2 \mathrm{~mm}$ diameter flat indenter. At rest, the elastic modulus for skin was $210 \mathrm{kPa}$, increasing to 446 and $651 \mathrm{kPa}$ under flexor and extensor loading of the muscle, respectively. Although in most studies the forearm is at rest during the experiments, in in vivo experiments on the volar forearm, the mechanical response at large length scales might be influenced by the tension of the underlying muscle.

\section{What value to use for the elastic modulus?}

To account for the multilayered and nonhomogeneous structure of the skin, as well as the relatively large deformations and any nonlinear effects in a relatively straightforward manner, it is suggested to use the effective elastic modulus $E_{\text {eff }}$, sometimes referred to as the apparent or equivalent elastic modulus. Although it might formally not be correct from a physical point of view, the concept of an effective elastic modulus is very useful in contact and friction modelling as it reduces a multiparameter expression to a single parameter. The effective elastic modulus is a combination of the respective moduli of the skin layers and of the underlying tissue such as muscles and bones. The relative contribution of each individual skin layer to the effective elastic modulus is determined by the ratio of the thickness of the layer $t$ to the length scale of the contact $a$. Generally, the effective elastic modulus can be expressed as

$$
E_{\text {eff }}=E_{\text {substrate }}+\left(E_{\text {layer }}-E_{\text {substrate }}\right) \Phi(z)
$$

in which $\Phi$ is a weight function depending on the relative penetration $z$. The relative penetration $z$ being defined as the ratio of the indentation depth 
to the layer thickness $\delta / t$, or as the ratio of the length scale of the contact to the film thickness $a / t$, which is actually a relative length scale. Several methods to determine the relation between the effective modulus and the moduli of which it is composed can be found in the literature. An analytical solution to calculate the effective modulus of a layered material developed by Swain and Menčik, ${ }^{29}$ based on the work of Gao et al. ${ }^{30}$ was used succesfully in the modelling of the contact between a rough surface and a layered material by Pasaribu and Schipper. ${ }^{31}$ However, this model is valid only for modulus ratios up to 2 . For a layered material such as the skin, the modulus ratios vary between 0.01 and 100, and the evolution of the effective elastic modulus with the ratio $a / t$ can be calculated following the numerical procedure of Perriot and Barthel. ${ }^{32}$ Based on the calculated results, they presented an empirical function $E_{\text {eff }}^{*}(a / t)$ for a singlelayer system. An analytical single-layer model developed by Bec et al. ${ }^{33}$ was used to analyse the elastic behaviour of skin by Pailler-Mattei et al., ${ }^{14}$ who described the effective elastic modulus of the skin by considering the skin as a soft elastic layer on a less compliant substrate, the muscle. To account for the relative contributions of the dermis and hypodermis they extended the Bec-model to a two-layer model. With this model, realistic values for the elastic moduli of the skin layers were obtained from the measured effective elastic modulus for relatively large length scales.

In the current study, the effects of the epidermis including the high modulus stratum corneum are incorporated by extending the two-layer model to a four-layer model. The global stiffness of the skin $K_{g}$ is given as the reciprocal sum of the stiffnesses of the different skin layers $K_{i}$ and of the underlying tissue $K_{n}$. From the definition of the stiffnesses $K_{i}=\pi a^{2} E_{i}^{*} / t$ and $K_{n}=2 E_{n}^{*} a$, the effective elastic modulus is given as

$$
\frac{1}{E_{e f f}^{*}}=2 \sum_{i=1}^{n-1} \frac{t_{i}}{f_{i}(a) \pi a E_{i}^{*}}+\frac{1}{f_{n}(a) E_{n}^{*}}
$$

To ensure correct boundary conditions, the polynomial functions $f_{i}(a)$ were introduced in the expressions of the different moduli. ${ }^{14,33}$

- When the contact size is very small as compared to the thickness of the upper layer, the effective elastic modulus equals the elastic modulus of the upper layer.

- If the properties of the different layers are the same as those of the substrate, the effective elastic modulus equals the elastic modulus of the substrate.

- If the properties of neighbouring layers are the same, those layers respond as one thick layer with the same elastic properties.

In analogy to the work of Bec et al. ${ }^{33}$ and PaillerMattei et al. ${ }^{14}$ for the four-layer model, this leads to the following expressions for the functions $f_{i}(a)$

$$
\begin{aligned}
f_{1}(a)= & 1+\frac{2 t_{1}}{\pi a} \\
f_{i}(a)= & \left(1+\frac{2}{\pi a} \sum_{j=1}^{i-1} t_{j}\right)\left(1+\frac{2}{\pi a} \sum_{j=1}^{i} t_{j}\right), \\
& \text { for } i=2, \ldots, n-1 \\
f_{n}(a)= & \left(1+\frac{2}{\pi a} \sum_{j=1}^{n-1} t_{j}\right)
\end{aligned}
$$

From equations (11) and (12) it can be seen that the relation between the effective elastic modulus of the skin $E_{e f f}^{*}$ and the size of the contact $a$ depends solely on the respective thicknesses $t_{i}$ and elastic moduli $E_{i}^{*}$ of the different skin layers, indicated by the subscripts $i=1, \ldots, 4$, and the underlying tissue indicated by the subscript $n=5$. Figure 4 gives a summary of the foregoing.

\section{Skin properties}

For the properties of the different skin layers, a wide range of values are reported in the literature. The surface of the skin is formed by the stratum corneum,

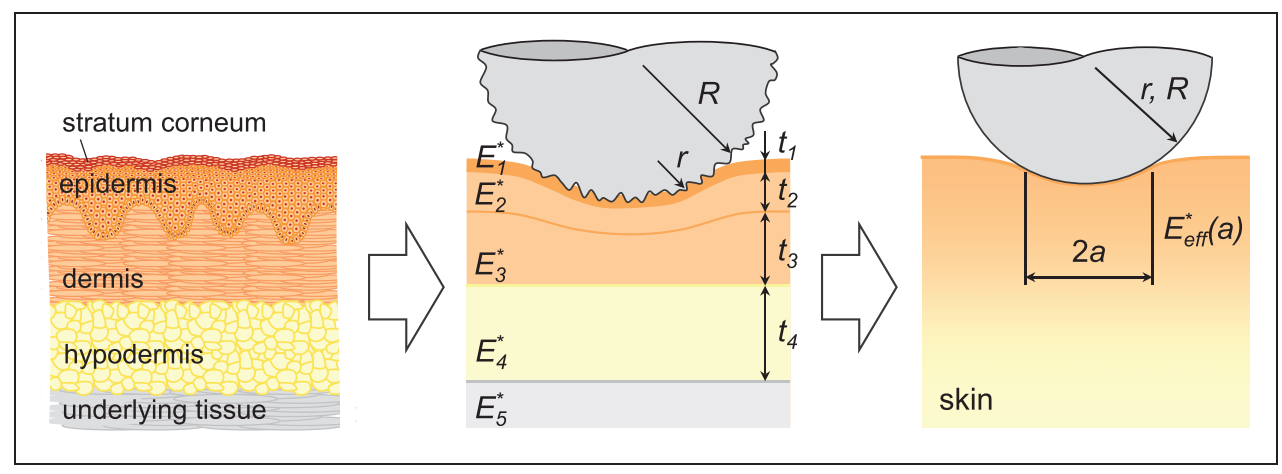

Figure 4. Schematic overview of the contact between a rough indenter and the skin simplified to the contact of a smooth spherical indenter and the skin. 
which is actually the outermost layer of the epidermis, the skin top layer. The thickness of the stratum corneum varies between 10 and $40 \mu \mathrm{m} .^{4,17,18}$ Values for the elastic modulus, measured from indentation experiments on isolated samples, vary from 3.5 to $2000 \mathrm{MPa}$ as shown in the section 'Mechanical properties of the skin'. For wet stratum corneum, the measured elastic modulus is considerably lower than for dry stratum corneum. ${ }^{4,17,18}$ The viable epidermis has a thickness varying from 40 to $150 \mu \mathrm{m} .^{4,9}$ and an elastic modulus of about $1.5 \mathrm{MPa} .{ }^{18}$ The second skin layer is the dermis, for which a thickness between 800 and $2000 \mu \mathrm{m}$ is reported. ${ }^{4,14}$ Elastic moduli are found ranging from 8.5 and $35 \mathrm{kPa}$ to $1.7 \mathrm{MPa} .{ }^{14,16}$ Below the dermis lies the hypodermis, or subcutaneous fat layer. The thickness of the hypodermis varies considerably with anatomical site and between persons. At the volar forearm, the thickness and elastic modulus are approximately $800 \mu \mathrm{m}$ and $2 \mathrm{kPa}$, respectively. ${ }^{14}$ Finally, for the underlying muscle, the elastic modulus is found to vary between 100 and $400 \mathrm{kPa} .^{14,28}$

Table 2 shows the parameter values for layer thickness $t_{i}$ and elastic modulus $E_{i}^{*}$ that have been used to calculate the effective elastic modulus of the skin of the volar forearm $E_{e f f}^{*}$ as a function of contact length scale $a$. Average values were calculated from the ranges found in the literature.

\section{Results and discussion}

Figure 5 shows the effective elastic modulus as a function of length scale calculated using the data from Table 2. The complex behaviour of the skin of the volar forearm can be characterised by a single curve describing the elastic behaviour as a function of the length scale of the contact. As discussed in the section 'Skin properties', different values for the respective layer thicknesses and elastic moduli can be found in the literature. The influence of the variation in these parameters on the effective elastic modulus has been indicated by dashed lines. The effective elastic modulus of the skin decreases several orders of magnitude when the length scale increases.

The evolution of the effective elastic modulus with contact length scale depicted in Figure 5 is the consequence of the rather complex anatomy of the skin. At different length scales different layers contribute to the apparent elasticity. The elastic moduli of the respective layers decrease with depth. With increasing length scale, the contribution of the lower and softer skin

Table 2. Parameters used for calculating the skin effective elastic modulus: average values and ranges (in parentheses).

\begin{tabular}{llllll}
\hline$i$ & Skin layer, tissue & & Elastic modulus $E_{i}^{*}(\mathrm{MPa})$ & Thickness $t_{i}(\mathrm{~mm})$ & References \\
\hline 1 & Stratum corneum & dry & $500(3.5-1000)$ & $0.025(0.01-0.04)$ & $4,17,18$ \\
& & wet & $30(10-50)$ & & $0.095(0.04-0.15)$ \\
2 & Viable epidermis & & 1.5 & $1.4(0.8-2)$ & $4,9,18$ \\
3 & Dermis & & $0.02\left(8-35 \times 10^{-3}\right)$ & $4,9,14,16$ \\
4 & Hypodermis & & $2 \times 10^{-3}$ & 0.8 & 14 \\
5 & Muscle & $0.25(0.1-0.4)$ & & 14,28 \\
\hline
\end{tabular}

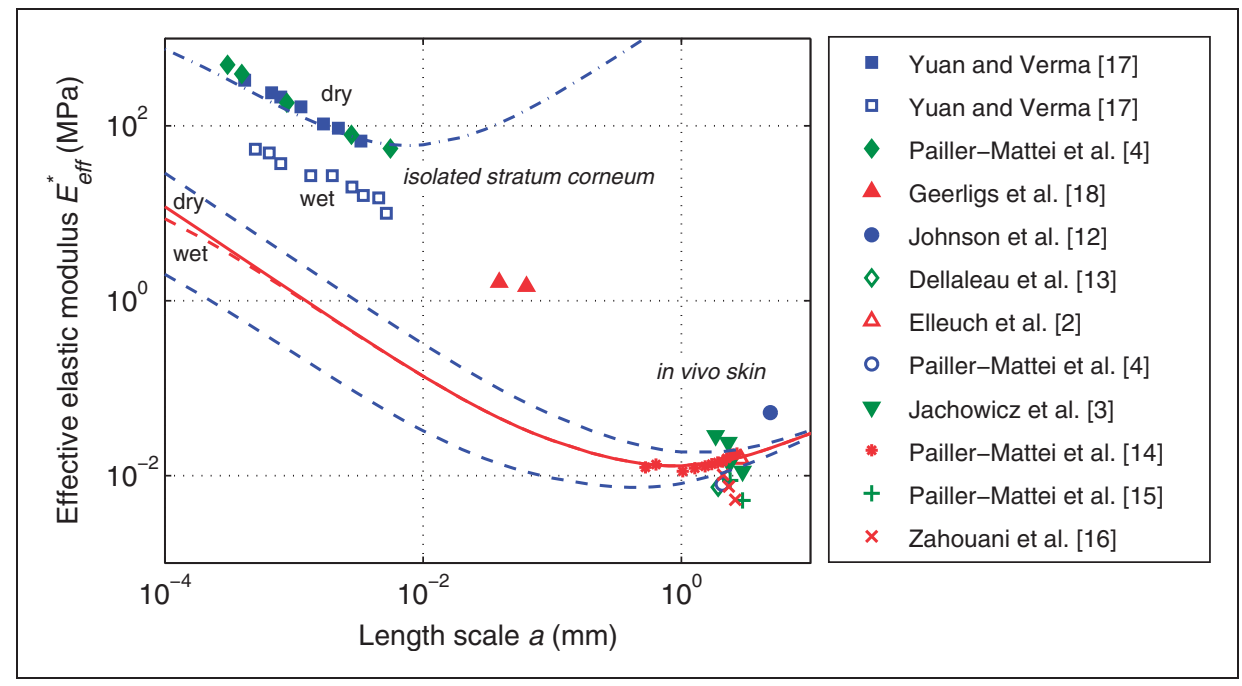

Figure 5. Effective elastic modulus of the skin of the volar forearm $E_{\text {eff }}^{*}$ as a function of length scale $a$ : calculated using average values (solid line), calculated using extreme values (dashed lines) and results from the literature. ${ }^{2-4,12-18}$ 
layers to the effective elastic modulus increases, its magnitude being determined by the ratio of elastically deformed volumes $a^{3} / t_{i} a^{2}$, or relative length scale $a / t_{i}$. Figure 6 plots the effective elastic modulus as a function of indentation depth $\delta$ for different indenter radii $R$ at indentations $\delta \leqslant 0.25 R$. At small indentation depths, the effective elastic modulus decreases with increasing indenter radius or, in other words, increasing elastically deformed volume due to the increasing contribution of the lower and softer skin layers. At larger indentation depths, the increasing contribution of the stiffer underlying tissue leads to an increase of the effective elastic modulus with increasing indenter radius.

\section{Stratum corneum and epidermis}

The stratum corneum serves a barrier function, preventing exogenous materials from penetrating into the body and vital components such as water from leaving the body. ${ }^{10}$ The stratum corneum is the outermost layer of the viable epidermis which consists of keratinocytes, viable cells which are migrating towards the outer surface, eventually forming the stratum corneum. This top layer is composed of cornified dead cells which have a diameter of $20-40 \mu \mathrm{m}$ and a thickness of $1 \mu \mathrm{m}$. The amount of keratin, a tough structural protein, ranges from $30 \%$ at the base layer of the epidermis to $80 \%$ in the stratum corneum. ${ }^{9}$ The corneocytes are held together by lipids and corneodesmosomes, protein structures acting as 'rivets'. The structure of the corneocytes being embedded in a intercellular lipid matrix is commonly referred to as a brick-and-mortar structure. ${ }^{34}$ From a mechanical point of view, the corneocytes provide the stratum corneum with rigidity, whereas the lipids give it, its flexibility.

Although in the studies described in the section 'Mechanical properties' of the skin, the elastic modulus is reported as a function of indentation depth, using equations (8) and (9) the reported values can be plotted as a function of the length scale of the contact. The elastic properties from stratum corneum obtained from the literature are plotted as a function of contact length scale in Figure 5. As compared to the high elastic modulus measured for isolated stratum corneum, the calculated effective elastic modulus at small length scales is rather low. Perriot and Barthel $^{32}$ calculated equivalent elastic moduli as a function of relative length scale $a / t$ for substrate to layer elastic moduli ratios ranging from $10^{-2}$ to $10^{2}$. Their results show that the mechanical response of the layered system is dominated by the softer material, whether the softer material is the layer or the substrate, which explains the difference observed from Figure 5. The proposed model can be applied to a soft layer supported by a harder substrate, i.e. $E_{n}^{*}=70 \mathrm{GPa}$, as indicated by the dashdotted line in Figure 5. Although it can be seen in Figure 5 that the elastic modulus of wet isolated stratum corneum is considerably lower than for dry stratum corneum. ${ }^{17}$ hydration has only a minor

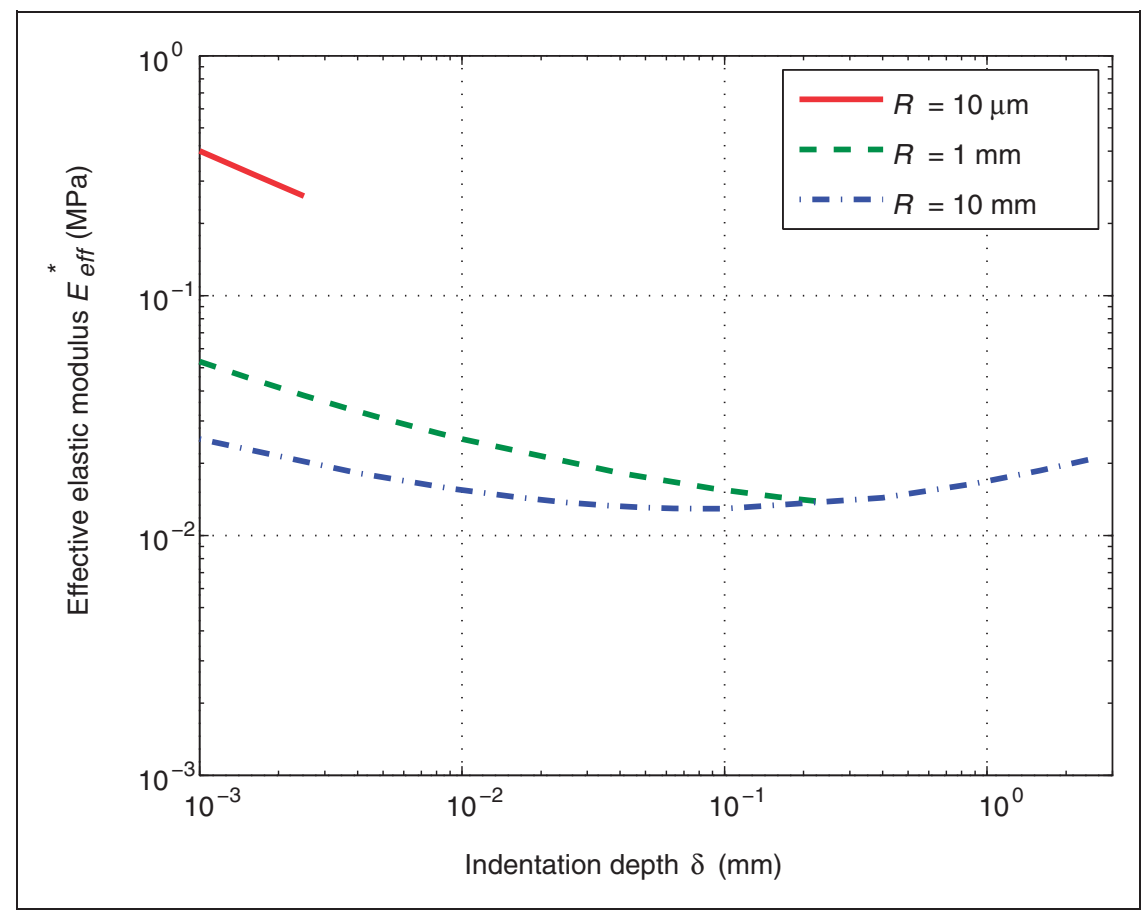

Figure 6. Effective elastic modulus of the skin of the volar forearm $E_{\text {eff }}^{*}$ as a function of indentation depth $\delta$ for different indenter radii $R$. 
influence on the magnitude of the effective elastic modulus.

When the length scale is of the same order as the thickness of the stratum corneum, the influence of the epidermis and lower skin layers increases and the effective elastic modulus is found to decrease due to the lower elasticity of these layers.

\section{Dermis and hypodermis}

The dermis forms the second layer of the skin. The dermis houses several of the skin's appendages, such as the hair follicles, sweat and sebaceous glands and different nerve cells. Adhesion between the dermis and the epidermis is provided by the dermal-epidermal junction appearing like fingerlike projections providing mechanical strength by increasing the contact area and by mechanical interlocking. The dermis is the main mechanical component of the skin, maintaining spatial organisation of the underlying tissues and protecting against mechanical stresses from the outside so acting as a shock absorber. It is composed of collagen and elastin fibers in a viscous fluid, the extrafibrillar matrix. The collagen is the load-carrying element with intramolecular crosslinks giving strength to the dermis. The elastin provides the skin with its elasticity. With age, the density and orientation of these fibers change, leading to a considerable decrease in elasticity.

Underlying the dermis is the hypodermis, or subcutis, connecting the dermis to the underlying skeletal components. The hypodermis houses bloodvessels and nerves. Furthermore, this subcutaneous fat layer provides for energy storage, thermal insulation and is a cushion, protecting against mechanical shock. The thickness of the hypodermis is $1 \mathrm{~mm}$ to $5 \mathrm{~cm}$ and depends strongly on anatomical site and other factors such as a person's diet. The hypodermis is an extremely viscous and soft layer.

The results for the elastic moduli measured in vivo are plotted as a function of contact length scale in Figure 5. Although the model omits the nonlinear characteristics of the skin and does not take into account the presence of hair follicles, the different glands and bloodvessels, the curve describes the evolution of the effective elastic modulus with the lenght scale of the contact rather well. Variation of properties of the lower and softer skin layers with age, gender or anatomical site give rise to the reported variations in elastic properties. Figure 7 shows the data reported earlier in Figures 1 and 5 for the reduced elastic modulus of in vivo human skin as a function of subject gender and age. With increasing age, the reduced elastic modulus at the larger length scales tends to decrease; for females, a lower elasticity is measured than for males. The variation in elastic properties can be contributed to a variation in elastic properties of the dermis.

After a decrease of several orders of magnitude with the length scale increasing from the micron to millimeter range, from a length scale of several millimeters a slight increase of the effective elastic modulus of the skin is caused by the underlying tissues such as muscle.

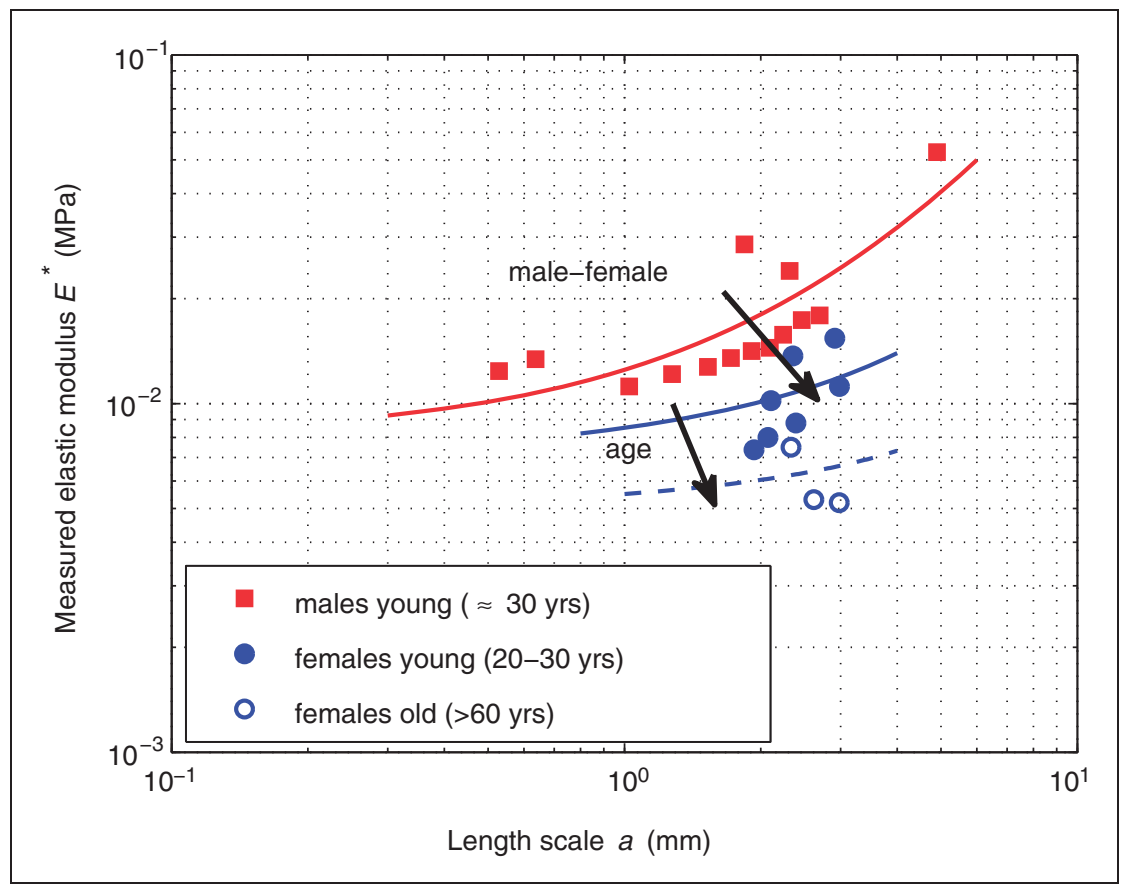

Figure 7. Measured elastic modulus of in vivo skin of the human volar forearm as a function of length scale. Data adapted from the literature. ${ }^{2-4,12-16}$ 


\section{Contact and friction}

As shown in equations (2) and (3), the friction force of a spherical probe sliding against the human skin is a function of the contact radius and the indentation depth. These contact parameters can be calculated iteratively from the indenter geometry and contact load using Hertz's equations (4) and (5) as depicted schematically in Figure 8. The effective elastic modulus of the skin decreases with increasing length scale to a minimum value, after which a slight increase can be observed. Obviously, the evolution of the effective elastic modulus with the length scale of the contact leads to a deviation from the relation predicted by Hertz, which assumes a constant elastic modulus. With decreasing elastic modulus, the contact radius shows a stronger increase with increasing indenter radius and contact load. The indentation depth shows a stronger increase with increasing contact load and, as it is inversely proportional to the indenter radius, a weaker decrease with increasing indenter radius.

Although the solution provided by Hertz is based on several assumptions ${ }^{5,6}$ Hertz's theory is commonly used in all contact problems involving spherical bodies. Dintwa et al..$^{35}$ investigated the accuracy of the Hertz model under conditions in which some of these assumptions were violated using the finite element method. They found that friction between the

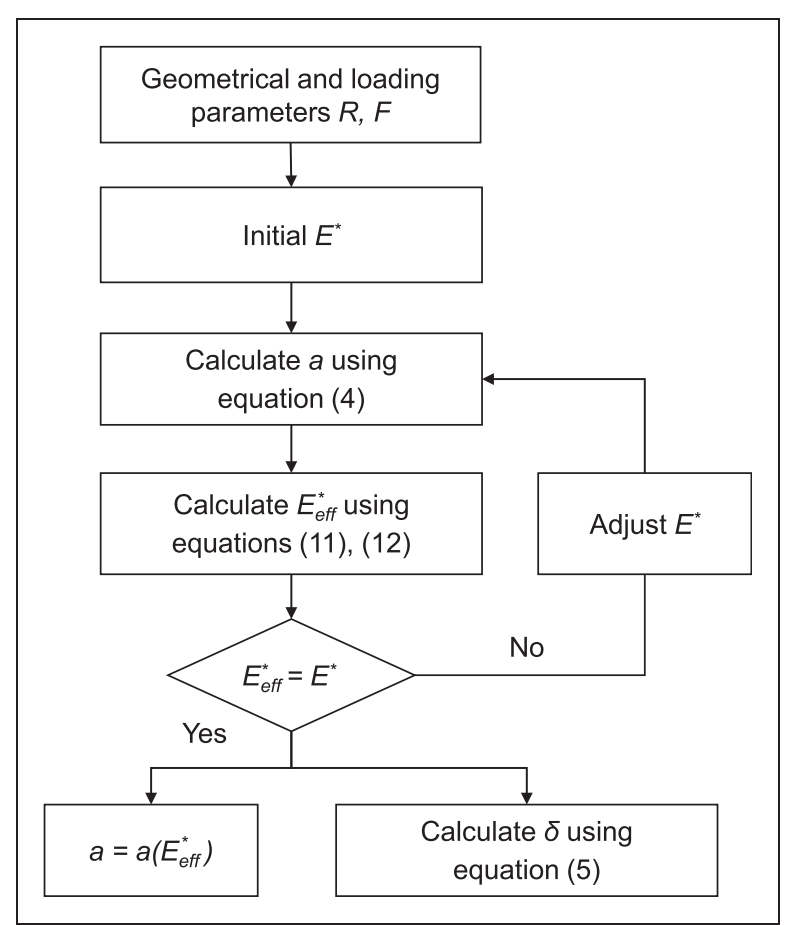

Figure 8. Algorithm for calculating the contact radius $a$ and indentation depth $\delta$ from the indenter radius $R$ and normal load $F$ taking into account the length scale effect of the effective elastic modulus $E_{\text {eff }}^{*}$ of the skin. contacting bodies (Hertz's theory assumes frictionless contacts) had no significant effect on the force-indentation relation predicted by Hertz, although the contact radius is slightly smaller. ${ }^{6}$ Furthermore, no significant errors were found when the contact surface is not flat, as is the case with a rigid body indenting the softer skin. However, at large indentations, the Hertz model appears to underestimate the normal force, the error increasing with increasing indentation. At a ratio $\delta / R=0.025$ an error of $9.2 \%$ was found. They proposed an equation for the correction of the Hertz model.

For a layered material the relation between contact radius and indentation as shown in equations (8) and (9), which are valid for homogeneous materials, break down. Perriot and Barthel ${ }^{32}$ calculated the contact force and indentation as a function of contact radius for the elastic contact of an axisymmetric indenter and a coated substrate. For substrate to layer elastic moduli ratios ranging from $10^{-2}$ to $10^{2}$, equivalent elastic moduli were calculated. For a spherical indenter they showed that the relation between indentation and contact radius is given by $\delta=\left(a^{2} / R\right) \cdot \Phi(a / t)$ in which $\Phi(a / t)$ deviates from one when the contact radius $a$ approaches the layer thickness $t$. For soft layers on an harder substrate, as in the experiments on isolated stratum corneum attached to a glass or metal substrate, $\Phi<1$. For large moduli ratio this leads to a maximum error of around $30 \%$.

Thus the curve depicted in Figure 5 can be used to estimate contact parameters of a spherical probe sliding against the nonglabrous skin of the inner forearm with reasonable accuracy. When data for the thickness and elastic modulus of the individual skin layers are known, the equations (11) and (12) can be used to predict the elastic behaviour at other anatomical sites. Contact models, such as the model proposed by $\operatorname{Archard}^{36}$ can be used to incorporate the role of the surface topography of the nonglabrous, or hairy, skin in the contact and friction behaviour. However, describing the contact behaviour of the glabrous skin of for example the fingerpad requires a more complex model than the one presented in this study, which was based on the sphere-on-flat geometry. Although at first sight the fingertip seems to approximate a soft sphere, its contact behaviour is not that simple, as was shown by Derler et al. ${ }^{37}$ They found that at low loads the pressure distribution showed a round profile corresponding to the distribution predicted by Hertzian theory. At larger loads, however, the pressure distribution was conical, which was attributed to the influence of the bony structure underneath the softer tissues of the finger pad. The contact and friction behaviour is further complicated by the particular surface texture of the fingerpad skin, the contact area being determined by the number of fingerprint ridges rather than by deformation of the ridges. ${ }^{38}$ 


\section{Conclusions}

Although an exact description of the mechanical behaviour of the skin requires an anisotropic, nonlinear, viscoelastic model, for contact modelling involving relatively small deformations, the mechanical behaviour can be described accurately by a single parameter: the effective elastic modulus. The complex behaviour of the skin of the volar forearm can be described using a closed-form expression $E=f(a)$ giving the elastic modulus $E$ as a function of contact length scale $a$. Data collected from the literature shows that the measured elastic modulus of the skin varies orders of magnitude with varying contact length scale. The calculated effective elastic modulus of the skin describes the evolution of the elastic modulus with contact length scale very well. The length scale of the contact, which in case of a spherical contact equals the contact radius $a$, is the parameter from which the effective elastic modulus is calculated. For a given load and geometry, geometrical parameters such as the contact radius and the indentation depth can be iteratively calculated using a set of analytical expressions.

The effective elastic modulus varies considerably with length scale. At the micro scale, for example when investigating the effect of textures or surface roughness, length scales are in the order of micrometers. The effective elastic modulus decreases several orders of magnitude with increasing length scale, from about $1 \mathrm{GPa}$ at the outer surface to $1 \mathrm{MPa}$ at a length scale of $1 \mu \mathrm{m}$. Although the elastic modulus of the stratum corneum decreases considerably with increasing hydration, there is only a minor influence of hydration on the magnitude of the effective elastic modulus. For indentation at the macro scale, for example in determining the apparent contact area in a tribological test, length scales are in the order of millimeters. The reduced elastic modulus is in the order of kilopascals, and varying with age and gender.

In this work a curve is presented, which describes the elastic response of the average inner forearm as a function of contact lenght scale. Because the expressions that were used to calculate the effective elastic modulus account for the elastic and geometrical properties of the skin layers, they can be used to predict the elastic behaviour at any other anatomical site for which these data are available. However, a study to confirm this was not conducted.

For the contact and friction modelling of human skin, a closed-form expression based on the anatomy of the skin exists, which yields the magnitude of the effective elastic modulus of the skin as a function of the length scale of the contact depending on variables such as age, gender and environmental conditions.

\section{Funding}

This research was carried out under project number M63.2.10409 in the framework of the Research Program of the Materials innovation institute M2i (www.m2i.nl).

\section{References}

1. Czichos H. Tribology: A systems approach to the science and technology of friction, lubrication and wear. Amsterdam: Elsevier, 1978.

2. Elleuch K, Elleuch R and Zahouani H. Comparison of elastic and tactile behavior of human skin and elastomeric materials through tribological tests. Pol Eng Sci 2006; 46(12): 1715-1720.

3. Jachowicz J, McMullen $\mathrm{R}$ and Prettypaul D. Indentometric analysis of in vivo skin and comparison with artificial skin models. Skin Res Technol 2007; 13(3): 299-309.

4. Pailler-Mattei C, Pavan S, Vargiolu R, et al. Contribution of stratum corneum in determining biotribological properties of the human skin. Wear 2007; 263(7-12): 1038-1043.

5. Hertz H. Über die Berührung fester elastischer Körper. $J$ Reine Angewandte Math 1882; 92: 156-171.

6. Johnson KL. Contact mechanics. Cambridge, UK: Cambridge University Press, 1999.

7. Hendriks CP and Franklin SE. Inuence of surface roughness, material and climate conditions on the friction of human skin. Tribol Lett 2009; 37(2): 361-373.

8. Van Kuilenburg J, Masen MA, Groenendijk MNW, et al. An experimental study on the relation between surface texture and tactile friction. Tribol Int 2012; 48: $15-21$.

9. Tobin DJ. Biochemistry of human skin - our brain on the outside. Chem Soc Rev 2006; 35(1): 52-67.

10. Subramanyan K, Misra M, Mukherjee S, et al. Advances in the materials science of skin: A composite structure with multiple functions. MRS Bulletin 2007; 32(10): 770-778.

11. Greenwood JA and Williamson JBP. Contact of nominally at surfaces. Proc $R$ Soc Lond A 1966; 295(1442): 300-319.

12. Johnson SA, Gorman DM, Adams MJ, et al. The friction and lubrication of human stratum corneum. In: CM Taylor and D Dowson (eds) Thin films in tribology, Proceedings of the 19th Leeds-Lyon symposium on tribology, University of Leeds, UK, vol. 25, 1993, pp.663-672.

13. Delalleau A, Josse G, Lagarde J-M, et al. Characterization of the mechanical properties of skin by inverse analysis combined with the indentation test. J Biomech 2006; 39(9): 1603-1610.

14. Pailler-Mattei $C, B e c S$ and Zahouani H. In vivo measurements of the elastic mechanical properties of human skin by indentation tests. Med Eng Phys 2008; 30(5): 599-606.

15. Pailler-Mattei C, Nicoli S, Pirot F, et al. A new approach to describe the skin surface physical properties in vivo. Colloids Surf B 2009; 68(2): 200-206.

16. Zahouani H, Pailler-Mattei C, Sohm B, et al. Characterization of the mechanical properties of a dermal equivalent compared with human skin in vivo by indentation and static friction tests. Skin Res Technol 2009; 15(1): 68-76.

17. Yuan Y and Verma R. Measuring microelastic properties of stratum corneum. Colloids Surf B 2006; 48(1): 6-12.

18. Geerligs M, van Breemen L, Peters G, et al. In vitro indentation to determine the mechanical properties of epidermis. J Biomech 2011; 44(6): 1176-1181. 
19. Adams MJ, Briscoe BJ and Johnson SA. Friction and lubrication of human skin. Tribol Lett 2007; 26(3): 239-253.

20. Oliver WC and Pharr GM. An improved technique for determining hardness and elastic modulus using load and displacement sensing indentation experiments. $J$ Mater Res 1992; 7(06): 1564-1583.

21. Wildnauer RH, Bothwell JW and Douglass AB. Stratum corneum biomechanical properties I. Influence of relative humidity on normal and extracted human stratum corneum. J Inv Dermatol 1971; 56(1): 72-78.

22. Park AC and Baddiel CB. Rheology of stratum corneum-I: A molecular interpretation of the stressstrain curve. J Soc Cosm Chem 1972; 23: 3-12.

23. Papir YS, Hsu K-H and Wildnauer RH. The mechanical properties of stratum corneum: I. The effect of water and ambient temperature on the tensile properties of newborn rat stratum corneum. Biochim Biophys Acta 1975; 399(1): 170-180.

24. Egawa M and Kajikawa T. Changes in the depth profile of water in the stratum corneum treated with water. Skin Res Technol 2009; 15(2): 242-249.

25. Nakagawa N, Matsumoto M and Sakai S. In vivo measurement of the water content in the dermis by confocal raman spectroscopy. Skin Res Technol 2010; 16(2): 137-141.

26. Wu KS, van Osdol WW and Dauskardt HR. Mechanical properties of human stratum corneum: Effects of temperature, hydration, and chemical treatment. Biomaterials 2006; 27(5): 785-795.

27. Flynn C, Taberner A and Nielsen P. Measurement of the force-displacement response of in vivo human skin under a rich set of deformations. Med Eng Phys 2011; 33(5): 610-619.

28. Iivarinen JT, Korhonen RK, Julkunen $\mathrm{P}$, et al. Experimental and computational analysis of soft tissue stiffness in forearm using a manual indentation device. Med Eng Phys 2011; 33: 1245-1253.

29. Swain MV and Menčik J. Mechanical property characterization of thin films using spherical tipped indenters. Thin Solid Films 1994; 253(1-2): 204-211.

30. Gao H, Chiu C-H and Lee J. Elastic contact versus indentation modeling of multi-layered materials. Int $J$ Sol Sruct 1992; 29(20): 2471-2492.

31. Pasaribu HR and Schipper DJ. Application of a deterministic contact model to analyze the contact of a rough surface against a at layered surface. J Tribol 2005; 127(2): 451 .

32. Perriot A and Barthel E. Elastic contact to a coated half-space: Effective elastic modulus and real penetration. J Mater Res 2004; 19(02): 600-608.

33. Bec S, Tonck A, Georges J-M, et al. Improvements in the indentation method with a surface force apparatus. Phil Mag A 1996; 74(5): 1061-1072.

34. Wu KS, Van Osdol W and Dauskardt HR. Mechanical and microstructural properties of stratum corneum. MRS Online Proceedings Library 2002; 724: 27-33.

35. Dintwa E, Tijskens E and Ramon H. On the accuracy of the Hertz model to describe the normal contact of soft elastic spheres. Granular Matter 2007; 10: 209-221.

36. Archard JF. Elastic deformation and the laws of friction. Proc Roy Soc Lond Ser A. Math Phys Sci 1957; 243(1233): 190-205.

37. Derler S, Süess J, Rao A, et al. Inuence of variations in the pressure distribution on the friction of the finger pad. Tribol Int, (in press).

38. Spurr RT. Fingertip friction. Wear 1976; 39(1): $167-171$.

\section{Appendix}

\section{Notation}

$a$

A

E

$E^{*}$

$E_{\text {eff }}^{*}$

$F$
$F$

$F_{\mu}$

$F_{\mu, a d h}$

$F_{\mu, \text { def }}$

$R$

$t$

z

$\alpha$

$\beta$

$\delta$

$v$

$\tau$ contact radius, length scale $(\mathrm{m})$

contact area $\left(\mathrm{m}^{2}\right)$

elastic modulus $(\mathrm{Pa})$

reduced elastic modulus $(\mathrm{Pa})$

effective elastic modulus $(\mathrm{Pa})$

normal load $(\mathrm{N})$

friction force $(\mathrm{N})$

friction force due to adhesive shear $(\mathrm{N})$

friction force due to deformation losses

(N)

radius of curvature $(\mathrm{m})$

layer thickness $(\mathrm{m})$

relative penetration $(-)$

angle $\left({ }^{\circ}\right)$

viscoelastic loss fraction (-)

indentation depth, deformation $(\mathrm{m})$

Poisson ratio $(-)$

shear strength of the interface $(\mathrm{Pa})$ 